\title{
Applying a Bayesian Approach to Identification of Orthotropic Elastic Constants from Full Field Displacement Measurements
}

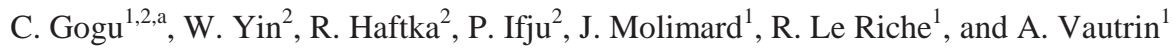 \\ ${ }^{1}$ Ecole des Mines de Saint Etienne, Centre Sciences des Matériaux et des Structures, 158 cours \\ Fauriel, 42023 Saint Etienne cedex 2, France \\ ${ }^{2}$ University of Florida, Mechanical and Aerospace Engineering Department, PO Box 116250, \\ Gainesville, FL 32611, USA
}

\section{Extended Abstract}

A major challenge in the identification of material properties is handling different sources of uncertainty in the experiment and the modelling of the experiment for estimating the resulting uncertainty in the identified properties.

Numerous improvements in identification methods have provided increasingly accurate estimates of various material properties. However, characterizing the uncertainty in the identified properties is still relatively crude. Different material properties obtained from a single test are not obtained with the same confidence. Typically the highest uncertainty is associated with respect to properties to which the experiment is the most insensitive. In addition, the uncertainty in different properties can be strongly correlated, so that obtaining only variance estimates may be misleading.

A possible approach for handling the different sources of uncertainty and estimating the uncertainty in the identified properties is the Bayesian method. This method was introduced in the late 1970s in the context of identification [1] and has been applied since to different problems, notably identification of elastic constants from plate vibration experiments [2]-[4]. The applications of the method to these classical pointwise tests involved only a small number of measurements (typically ten natural frequencies in the previously cited vibration test) which facilitated the application of the Bayesian approach.

For identifying elastic constants, full field strain or displacement measurements provide a high number of measured quantities (one measurement per image pixel) and hence a promise of smaller uncertainties in the properties. However, the high number of measurements represents also a major computational challenge in applying the Bayesian approach to full field measurements.

To address this challenge we propose an approach based on the proper orthogonal decomposition (POD) of the full fields in order to drastically reduce their dimensionality. POD is based on projecting the full field images on a modal basis, constructed from sample simulations, and which can account for the variations of the full field as the elastic constants and other parameters of interest are varied. The fidelity of the decomposition depends on the number of basis vectors used. Typically even complex fields can be accurately represented with no more than a few dozen modes and for our problem we showed that only four or five modes are sufficient [5].

\footnotetext{
a e-mail : christian.gogu@gmail.com
} 
To further reduce the computational cost of the Bayesian approach we use response surface approximations of the POD coefficients of the fields. We show that $3^{\text {rd }}$ degree polynomial response surface approximations provide a satisfying accuracy. The combination of POD decomposition and response surface methodology allows to bring down the computational time of the Bayesian identification to a few days.

The proposed approach is applied to Moiré interferometry full field displacement measurements from a traction experiment on a plate with a hole. The laminate with a layup of [45,$45,0]_{\mathrm{s}}$ is made out of a Toray ${ }^{\circledR}$ T800/3631 graphite/epoxy prepreg. The measured displacement maps are provided in Figure 1.
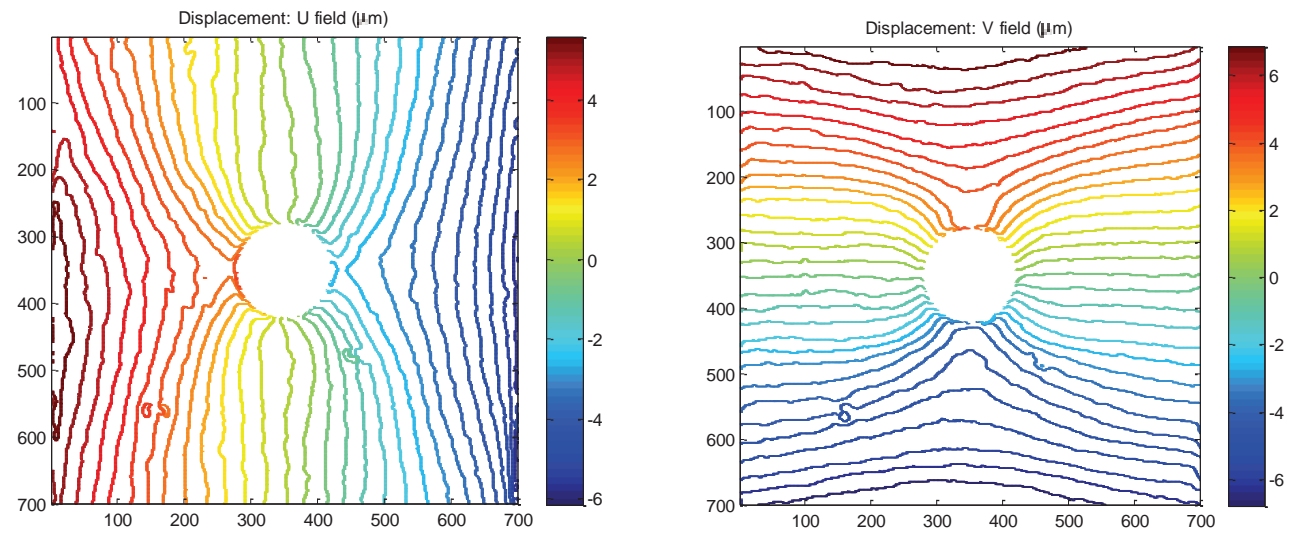

Fig. 1. Displacement maps measured by Moiré interferometry.

The mean values of the identified properties joint probability density function are in agreement with previous identifications carried out on the same material. Furthermore the probability density function also provides the coefficient of variation with which the properties are identified as well as the correlations between the various properties. We find that while the longitudinal Young's modulus is identified with good accuracy (low standard deviation), the Poisson's ration is identified with much higher uncertainty. Several of the properties are also found to be correlated. The identified uncertainty structure of the elastic constants (i.e. variance co-variance matrix) has potential benefits to reliability analyses, by allowing a more accurate description of the input uncertainty.

An additional advantage of the Bayesian approach is that it provides a natural way (in the form of the prior probability density function) for accounting for prior information that may be available on the material properties thought. This is of great interest for reducing the uncertainty on properties that can only be determined with low confidence from the plate with a hole experiment, such as Poisson's ratio or transverse Young's modulus in our case.

\section{References}

1. Isenberg, J., Proc. of ASME Design Engineering Technical Conferences, (1979)

2. Sol, H., Identification of anisotropic plate rigidities, $\mathrm{PhD}$ dissertation (Vrije Universiteit Brussel, 1986)

3. Lai, T.C. and Ip, K.H., Composite Structures, 34, (1996)

4. Gogu, C., Haftka, R. T., Le Riche, R., Molimard, J., Vautrin, A., Sankar, B. V., Proc. $11^{\text {th }}$ AIAA Non-Deterministic Approaches Conference, Palm Springs, CA, (May 2009)

5. Gogu, C., Haftka, R. T., Le Riche, R., Molimard, J., Vautrin, Proc. $17^{\text {th }}$ International Conference on Composite Materials, Edinburgh, UK, (July 2009) 